\title{
The Two-Nucleon System in Effective Field Theory: Searching for the Power Counting
}

\author{
M. Pavón Valderrama*t \\ Instituto de Física Corpuscular (IFIC), Centro Mixto CSIC-Universidad de Valencia, \\ Institutos de Investigación de Paterna, Apartado 22085, E-46071 Valencia, Spain. \\ Institut de Physique Nucléaire, Université Paris-Sud, IN2P3/CNRS, F-91406 Orsay Cedex. \\ E-mail: pavonvalderrama@ipno.in2p3.fr
}

\begin{abstract}
Deriving nuclear forces from quantum chromodynamics is amongst the most important challenges of nuclear physics. The aim of the effective field theory formulation of nuclear forces is to bridge this gap through chiral symmetry breaking, the main low energy manifestation of quantum chromodynamics. However, the realization of this goal is not automatic. The effective field theory we are using must be renormalizable. Only if this condition is met can we really link the low energy dynamics of pions and nucleons with that of the the quarks and gluons conforming them. But, apart from the theoretical motivation, there is a more practical downside of renormalizability. If we have this property, calculations of observables in nuclear physics become systematic and, in particular, we can know the error of theoretical predictions in advance. This property is known as power counting. The original formulation of nuclear effective field theory due to Weinberg - the Weinberg counting -, though inspired and ingenious, failed to implement renormalizability and hence power couting. In this contribution we will explain what is missing in the Weinberg counting and how to improve on it to guarantee a fully consistent effective field theory for two-nucleon processes. As a result we will arrive at a theory in which contact range interactions - that is, four nucleon vertices - play a more prominent role than expected in Weinberg's original proposal.
\end{abstract}

The 7th International Workshop on Chiral Dynamics,

August 6 -10, 2012

Jefferson Lab, Newport News, Virginia, USA

\footnotetext{
*Speaker.

$\dagger$ This work has been supported by the DGI under contract FIS2011-28853-C02-02, the Generalitat Valenciana contract PROMETEO/2009/0090, the Spanish Ingenio-Consolider 2010 Program CPAN (CSD2007-00042) and the EU Research Infrastructure Integrating Initiative HadronPhysics2.
} 
Nowadays one of the most fascinating theoretical problems of nuclear physics is the explanation of nuclear forces in terms of quantum chromodynamics (QCD). Though there are plenty of excellent descriptions of the nuclear force, they are commonly phenomenological and as such lack a clear connection with QCD. To remedy this situation we can try the strategy of computing the nuclear force directly in the lattice. Explorations in this line are however still preliminary and have not reached the physical pion mass yet $[1,2]$. Alternatively the effective field theory (EFT) formulation of nuclear forces provides a more indirect path to link nuclear physics with QCD [3]. For that, the EFT approach incorporates the main low energy manifestation of QCD - broken chiral symmetry -, which constrains in a very particular way the pion-nucleon dynamics. In addition, due to renormalizability, chiral EFT can be considered to be equivalent to low energy QCD. The reason is that chiral EFT corresponds to the renormalization group evolution of QCD in the infrared limit ${ }^{1}$. As a consequence of renormalizability EFTs contain a peculiar feature known as power counting, which means that all the possible interactions among the low energy degrees of freedom of the theory can be ordered from more to less relevant. This entails a remarkable simplification in the calculation of observables in nuclear physics: if we want to achieve a certain level of accuracy in theoretical predictions, we know in advance what to include and what to neglect in the computations. Probably, this is the main practical advantage of nuclear EFT over the traditional approach. At this point it is fundamental to remind that the prerequisite for all these desirable features is renormalizability, something that is forgotten more often than not.

However the application of the EFT concept to nuclear physics is far from trivial. The reason is that nuclear physics is non-perturbative. In the two-nucleon sector for instance we have two states that are located almost at the threshold, the deuteron and the virtual state in the singlet. In contrast the traditional knowledge of renormalizability in EFTs is perturbative (see e.g. Ref. [4] for a lucid exposition) and not easily adapted to the nuclear case. Thus, when applying the EFT concept for the first time in the few nucleon sector Weinberg proposed $[5,6]$ an interesting workaround: though nuclear physics is non-perturbative, nuclear forces can be expressed as a perturbative series in chiral EFT. In other words, we know how renormalization works for the two-nucleon potential. The natural thing to do then is to concentrate on the potential and derive all observables quantities from it, as has always been the case in traditional nuclear physics. The idea works as follows: first we begin by noticing that in the EFT formulation the two-nucleon potential can be expanded as a series of contributions of diminishing importance at low energies. Graphically we have:

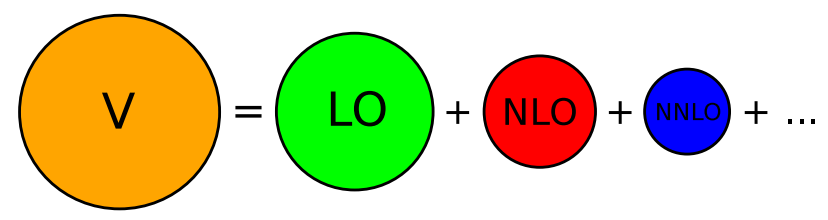

where LO stands for leading order, NLO for next-to-leading order and NNLO for next-to-next-toleading order, and the dots indicate higher order contributions that are expected to be very small at low energies. Each of the blobs in the figure above represents a set of Feynman diagrams involving nucleons and pions, while the size of the blob indicates their relative importance at low energies.

\footnotetext{
${ }^{1}$ Of course the practical problem remains of how to unconver the detailed relationship between chiral EFT and QCD, something that could be probably done in the lattice in the near future.
} 
Next we iterate this potential in the Schrödinger or Lippmann-Schwinger equation to obtain scattering amplitudes, i.e. the T-matrix. The natural expectation in the Weinberg counting is that, even if we do not know the inner workings of non-perturbative renormalization, the T-matrix will follow the same power counting as the potential, that is:

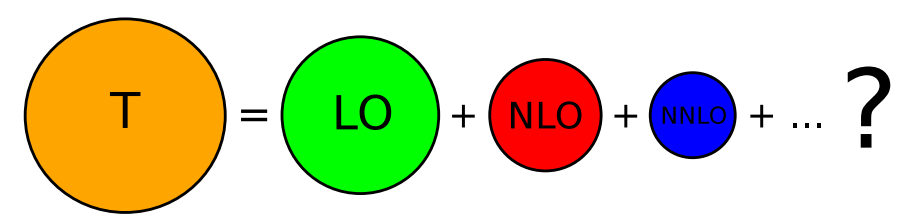

where I have put the interrogation mark to indicate that we do not really know for sure. Though a sensible expectation, there is a catch, as we will see. As a historical note it is worth mentioning that the Weinberg counting has been followed with enthusiasm in the literature and eventually, at high enough order ( $\mathrm{N}^{3} \mathrm{LO}$, i.e. next-to-next-to-next-to-leading order), it has been able to produce nucleon-nucleon potentials that can describe the two-nucleon scattering data at $E_{\text {lab }} \leq 300 \mathrm{MeV}$ with a $\chi^{2} /$ d.o.f. $\sim 1[7,8]$.

What can fail then in the Weinberg counting? Well, the problem is that power counting only works at low energies. Yet, we are iterating all the EFT contributions to the potential regardless of their order. As a consequence of the iteration process, we will have two-nucleon loops that are going to probe the high energy structure of the potential, where the EFT description is no longer valid. At high enough energies power counting breaks down and each new contribution to the chiral potential is not necessarily smaller than the previous one. I illustrate this point in figure 1, where I show the nucleon-nucleon EFT potential at long and short distances. As can be seen, power counting works as expected at long distances. But in contrast, at short distances the situation is the opposite: each new piece of the potential is bigger than the previous one. In pictures:

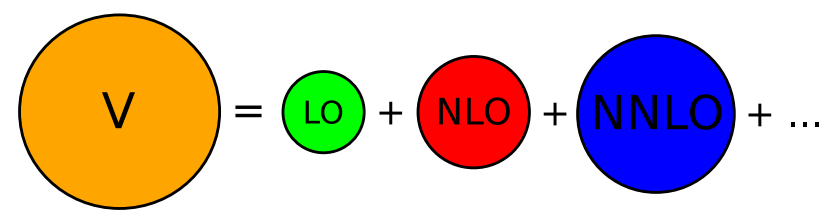

and if we do not do take precise measures to counterbalance the short distance physics probed in the loops, power counting will most probably be lost at the level of the T-matrix.

The solution to this problem involves the renormalization of the scattering amplitude. This requires the following steps: first we cut off the loop momentum to avoid probing the unphysical short range structure of the EFT potentials. But this is not enough: the regularization of the loops at $\Lambda$ - the cut-off scale - causes the observable quantities to depend on $\Lambda$, destroying the predictive power of the theory. Thus, as a second step, we include four nucleon vertices - counterterms - to absorb the cut-off dependence. At this point it is worth mentioning that the Weinberg counting is expected to be used with a finite cut-off and already contains counterterms. But even so it fails to be renormalizable. The counterterms that are expected in the Weinberg counting are not able to absorb the fairly strong cut-off dependence generated by the EFT potential, as has been repeatedly proven in a series of works $[9,10,11]$ that analyze the renormalizability of the Weinberg scheme. The conclusion is that we need to modify and correct the Weinberg counting. 

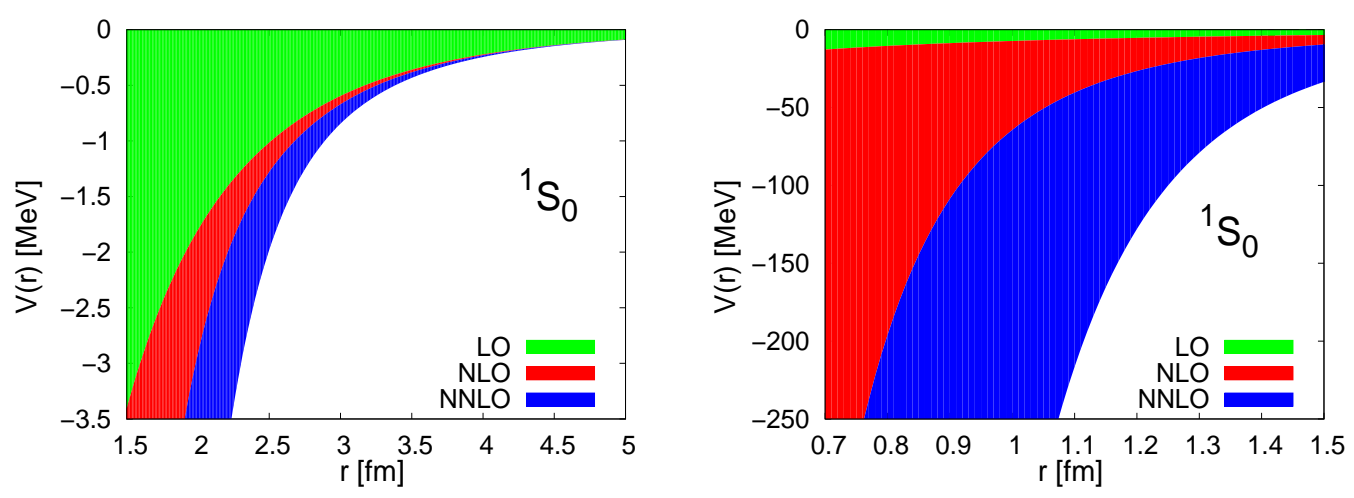

Figure 1: The chiral two-nucleon potential at LO, NLO and NNLO in the ${ }^{1} S_{0}$ channel. In the left panel (a), we show the contributions to the potential at long distances (between 1.5 and $5 \mathrm{fm}$ ). The bands represent the size of the contribution at each order: we can appreciate that at long distances the size of the contributions diminish with the order, In the right panel (b), we can see how the pattern breaks down at short enough distances (in particular, between 0.7 and $1.5 \mathrm{fm}$ ), in which case power counting no longer works as expected and each new contribution is bigger than the previous one.

Actually, the way to work out a proper power counting for the two-nucleon system is straightforward. It only requires to follow a small number of steps, like a recipe. They are the following:

a) We only iterate the leading order piece of the potential, which can be defined as the diagrams that happen to be more important than all the others at low energy.

b) The subleading order pieces of the potential - the diagrams that we have not included in the previous step - enter as perturbations (they are small corrections after all).

c) At every step in the calculation, we check for the cut-off independence of the results. If this condition is not fulfilled, we include new counterterms to absorb the divergences.

There is one thing left to decide: which is the leading order piece of the potential? The answer is not difficult: we include the one pion exchange potential plus a few contact range interactions in the S-, P-waves and optionally D-waves to make scattering amplitudes renormalizable, as discussed in Ref. [9]. Now, the next thing to do is to include the subleading order pieces of the potential and the counterterms. Determining the necessary number of counterterms is a problem that can be elucidated by several means, such as renormalization group analysis [12] and the perturbative renormalizability of the amplitudes in coordinate $[14,15]$ and momentum space $[16,17,18]$. The outcome of all these approaches is extremely similar at the fundamental level. However when one looks at the fine print there are subtle differences among them, a point that should be clarified in subsequent works hopefully for the relief of power counting aficionados and connoisseurs. Yet I feel that these differences are merely a manifestation of the possibility of reordering the power counting expansion in different ways: one can probably rearrange the EFT expansion without altering the convergence properties of the series, just as happens with a standard power series.

Finally, the comparison with the data (or, equivalently, with the high-precision potentials [13]) is remarkably good. In Figure 2 one can see a selection of the results for S- and P-waves at LO, NLO and NNLO. The phase shifts obtained within a nuclear EFT with explicit power counting [14, 

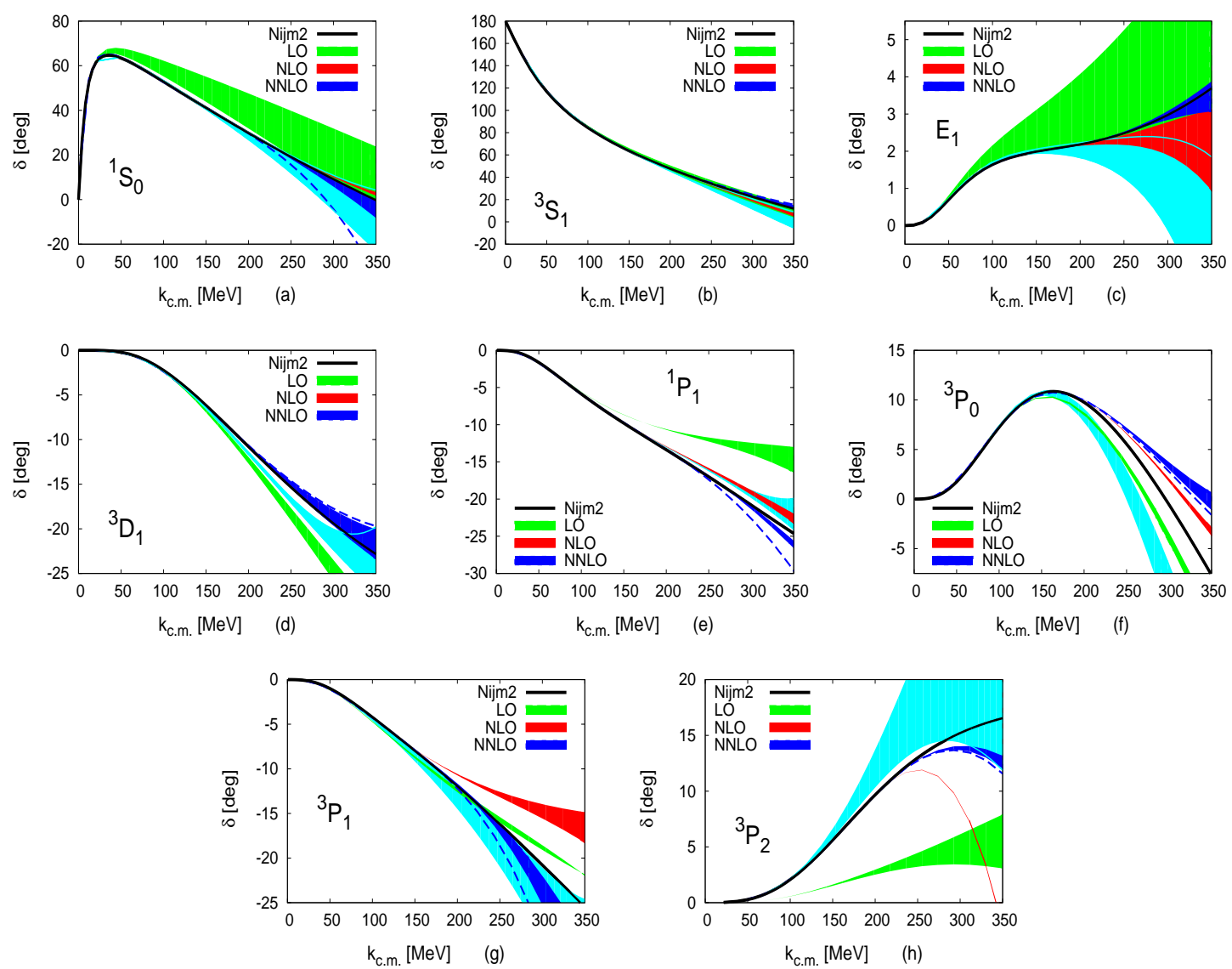

Figure 2: The S- and P-wave phase shifts in two-nucleon scattering for nuclear EFT, where $\delta$ is the phase shift and $k_{\text {c.m. }}$. the center-of-mass momentum. They have been calculated following the power counting described in the present contribution, where the one pion exchange potential is non-perturbative and two pion exchange is included as a perturbation $[14,15]$. The bands reflect the cut-off uncertainty of the results in the range $r_{c}=0.6-0.9 \mathrm{fm}$ (boundary radius). The dashed line (not always visible) represents the the $0.3 \mathrm{fm}$ NNLO results. The phase shifts are compared with the corresponding ones in the Weinberg counting (the light blue band in the figures), which are calculated from the NNLO potential of Refs. [19, 20].

15] are actually better than the corresponding ones in the Weinberg scheme when computed at the same order [19, 20]. In hindsight this is not surprising: power counting at the level of the scattering amplitudes requires a larger number of counterterms, i.e. free parameters, than what is expected in naive dimensional analysis. For the partial waves shown in Figure 2 at NNLO we have three counterterms in the ${ }^{1} S_{0}$ phase, two (per phase) in the ${ }^{3} S_{1}, E_{1},{ }^{3} D_{1},{ }^{3} P_{0}$ and ${ }^{3} P_{2}$ and one in the ${ }^{1} P_{1}$ and ${ }^{3} P_{1}$, while in Weinberg we have two per $\mathrm{S}$-wave, one for the $\mathrm{S}$ - to $\mathrm{D}$-wave mixing and one per P-wave. I said in hindsight as the statement is only obvious now that we know that chiral two pion exchange can be tamed in perturbation theory, a non-trivial result from the point of view of conventional wisdom. In the near future we expect to extend actual power counting to chiral extrapolations, electromagnetic reactions on the deuteron and the three-nucleon sector. In doing so we will further put to the test the usefulness of the nuclear EFT approach. 


\section{References}

[1] S. R. Beane, P. F. Bedaque, K. Orginos and M. J. Savage, Phys. Rev. Lett. 97, 012001 (2006) [hep-lat/0602010].

[2] N. Ishii, S. Aoki and T. Hatsuda, Phys. Rev. Lett. 99, 022001 (2007) [nucl-th/0611096].

[3] R. Machleidt and D. R. Entem, Phys. Rept. 503, 1 (2011) [arXiv:1105.2919 [nucl-th]].

[4] J. Polchinski, Nucl. Phys. B 231, 269 (1984).

[5] S. Weinberg, Phys. Lett. B 251, 288 (1990).

[6] S. Weinberg, Nucl. Phys. B 363, 3 (1991).

[7] D. R. Entem and R. Machleidt, Phys. Rev. C 68, 041001 (2003) [nucl-th/0304018].

[8] E. Epelbaum, W. Glöckle and U. -G. Meißner, Nucl. Phys. A 747, 362 (2005) [nucl-th/0405048].

[9] A. Nogga, R. G. E. Timmermans and U. van Kolck, Phys. Rev. C 72 (2005) 054006 [nucl-th/0506005].

[10] M. Pavon Valderrama and E. Ruiz Arriola, Phys. Rev. C 74, 054001 (2006) [nucl-th/0506047].

[11] M. Pavon Valderrama and E. Ruiz Arriola, Phys. Rev. C 74, 064004 (2006) [Erratum-ibid. C 75, 059905 (2007)] [nucl-th/0507075].

[12] M. C. Birse, Phys. Rev. C 74, 014003 (2006) [nucl-th/0507077].

[13] V. G. J. Stoks, R. A. M. Klomp, C. P. F. Terheggen and J. J. de Swart, Phys. Rev. C 49, 2950 (1994) [nucl-th/9406039].

[14] M. Pavon Valderrama, Phys. Rev. C 83, 024003 (2011) [arXiv:0912.0699 [nucl-th]].

[15] M. Pavon Valderrama, Phys. Rev. C 84, 064002 (2011) [arXiv:1108.0872 [nucl-th]].

[16] B. Long and C. J. Yang, Phys. Rev. C 84, 057001 (2011) [arXiv:1108.0985 [nucl-th]].

[17] B. Long and C. J. Yang, Phys. Rev. C 85, 034002 (2012) [arXiv:1111.3993 [nucl-th]].

[18] B. Long and C. J. Yang, Phys. Rev. C 86, 024001 (2012) [arXiv:1202.4053 [nucl-th]].

[19] E. Epelbaum, W. Glöckle and U. -G. Meißner, Eur. Phys. J. A 19, 125 (2004) [nucl-th/0304037].

[20] E. Epelbaum, W. Glöckle and U. -G. Meißner, Eur. Phys. J. A 19, 401 (2004) [nucl-th/0308010]. 